\title{
Viscosity of carbon nanotubes water based nanofluids: Influence of concentration and temperature
}

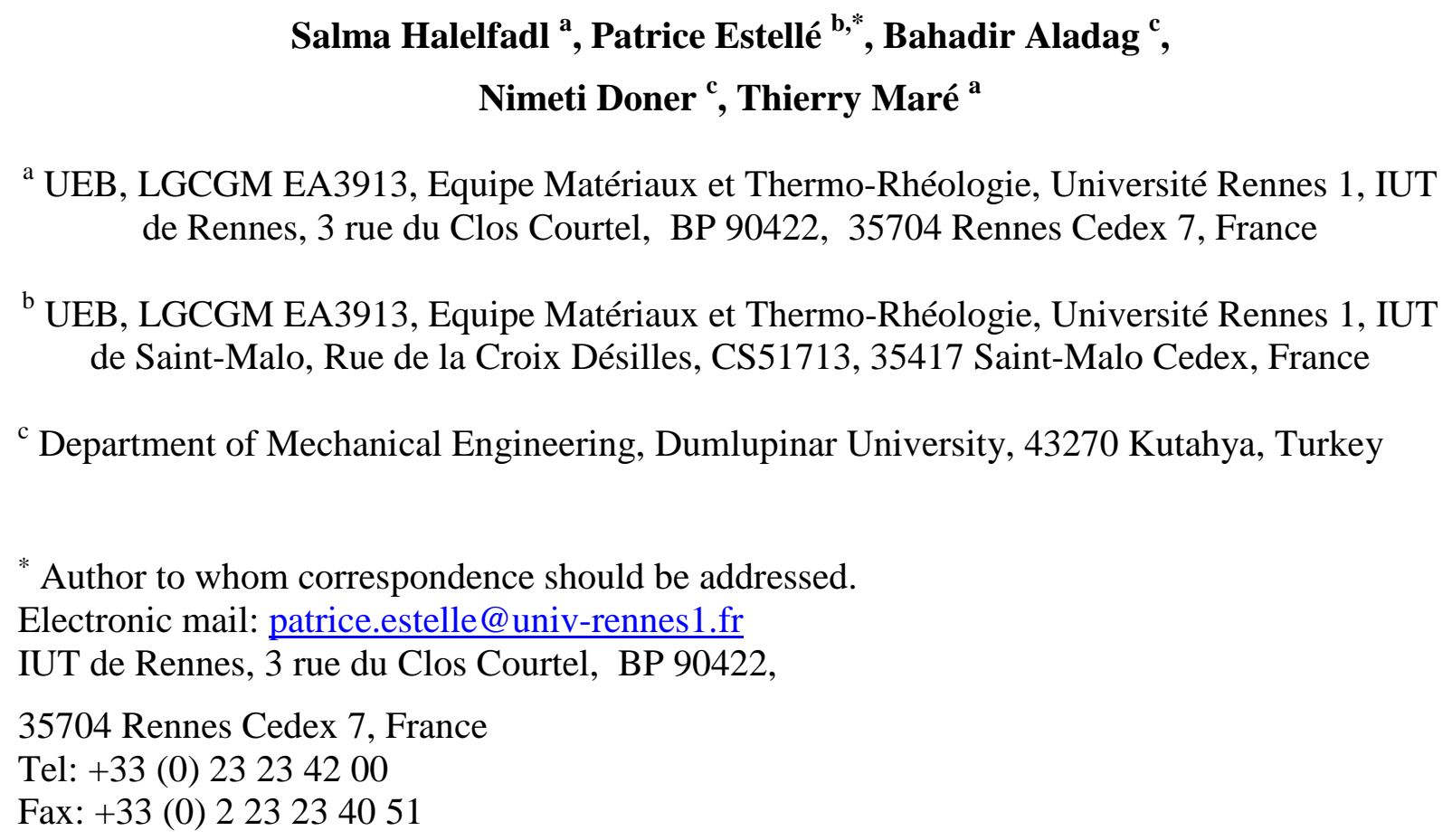

Abstract:

Experimental results on the steady state viscosity of carbon nanotubes water-based nanofluids are presented considering the influence of particle volume fraction and temperature ranging from 0 to $40^{\circ} \mathrm{C}$. The suspensions consist of multi-walled carbon nanotubes dispersed in deionized water and they are stabilized by a surfactant. The aspect ratio of nanotubes is close to 160 and the particle volume fraction varies between $0.0055 \%$ and $0.55 \%$. It is shown that the nanofluids behave as shear-thinning materials for high particle content. For lower particle content, the nanofluids are quite Newtonian. It is also observed that the relative viscosity of nanofluids at high shear rate does not vary with temperature. Moreover, the evolution of relative viscosity at high shear rate is well predicted by the Maron-Pierce model considering the effect of nanoparticles agglomerates.

Keywords: viscosity, CNT nanofluid, shear-thinning, agglomerates, temperature 


\section{Nomenclature}

L length of nanotubes

$\mathrm{d}$ average diameter of nanotubes

$r$ aspect ratio of nanotube, with $r=L / d$

$\mu_{\mathrm{bf}}$ viscosity of base fluid

$\mu_{\mathrm{nf}}$ viscosity of nanofluid

$\eta$ intrinsic viscosity

$\mu_{\mathrm{r}}$ relative viscosity

$\phi$ nanoparticle volume fraction

$\phi_{\mathrm{m}}$ maximum packing volume fraction

$\phi_{\mathrm{a}}$ effective volume fraction of aggregates a radius of nanoparticles

$56 \mathrm{a}_{\mathrm{a}}$ effective radii of aggregates

D fractal index

\section{Introduction}

60

Nanofluids are colloidal suspensions containing nanometer-sized particles of metals, oxides, carbides, nitrides, or nanotubes dispersed in a base fluid. The base fluid is usually a conventional fluid such as water, glycol, ethylene glycol, engine oil, etc. Over the past decade, nanofluids have attracted much interest owing to their high thermal conductivity and thermal performance compared to base fluids [1-6]. The thermal enhancement effects and the viscosity of nanofluids are strongly dependent on particle size and concentration, the nature of the base fluid, temperature and the presence of nanoclusters.

From a practical point of view, the viscosity of nanofluids is an important property for applications involving fluid flow and it is used to calculate the required pumping power. The viscosity can change due to the addition of solid nanoparticles and can cause the increase of pressure drops, affecting the efficiency of energy systems. It is also closely related to the stability and the structure of solid nanoparticles. The nanoparticle can agglomerate even at low concentration and also in the presence of a surfactant. In addition, it is well established that the shear-thinning behavior of nanofluids is mainly associated with the shape of nanoparticles and it is enhanced with the presence of nanoparticles agglomerates [7-11]. 
Carbon nanotubes (CNTs) nanofluids have attracted much attention because of their remarkable properties. Indeed, it was proved that carbon nanotubes nanofluids have a high thermal conductivity, high electrical conductivity $[12,13]$ and efficient mechanical properties [14]. The preliminary efforts were to treat and modify the surface of the CNTs to improve their solubility and to investigate the effect of surfactant and methods to disperse the nanotubes [15-18]. Nasiri et al. [18] have shown that the CNT structure and stability are strongly dependant on the functionalization and preparation methods of the nanosuspensions. The effect of chitosan as a dispersing agent on the viscosity of multi-wall carbon nanotubes (MWCNTs) dispersed in water was investigated by Phuoc et al. [19]. They showed that the rheological behavior of nanofluids is affected by both the quantity of chitosan and the CNT particle content.

Garg et al. [20] studied the effect of dispersing energy (ultra-sonication) on the viscosity of MWCNT aqueous-based nanofluids. They showed that these nanofluids exhibit a shear thinning behavior which is not related to the presence of the surfactant used. They have also shown that sonication time is first associated with declustering of nanoclusters CNT nanofluid. Then, increasing the sonication time breaks the nanotubes leading to less marked shear-thinning behavior of the nanofluid because of the shorter sizes of the nanotubes. Yang et al. [21] have also shown the effects of frequency and time of ultrasonication on agglomerate size and aspect ratio of nanotubes dispersed in oil. They reported that the aspect ratio of the nanotubes decreases both the dispersing time and the energy increase, resulting in less viscous nanosuspensions. The shear-thinning behavior of CNT nanofluids was also observed by Kanagaraj et al. [22]. They have investigated the rheological behavior of CNT nanofluid under a CNT weight fraction of $1 \%$ and for a temperature range of $20{ }^{\circ} \mathrm{C}$ to $50^{\circ} \mathrm{C}$ and shear rate ranging from 0 to $1000 \mathrm{~s}^{-1}$. Rheological study of Ponmozhi et al [23] has also demonstrated the shear-thinning behavior of CNT water-based nanofluids and the viscosity rise with CNT volume concentration at fixed shear rate and temperature. It was also shown that CNT water-based nanofluids can behave as a shear time dependent material due to the time dependency of agglomeration and disagglomeration kinetics under shear, which is linked to the structural network of the nanofluids [24]. Hence, the rheological behavior of CNT nanofluids can evolve following its preshear history [25]. The effects of base fluid and concentration on the rheological behavior of MWCNTs were investigated by Chen et al. [26]. They observed a Newtonian behavior for the studied MWCNTs dispersed in silicone oil, glycerol or water for all concentrations and temperatures. They also reported the effect of temperature; the viscosity of nanofluids decreases when the temperature increases. 
110 As far as we know, there are few reports of the rheological properties of aqueous CNT 111 nanofluids. To date, no attempts have been made to interpret the rheological data of such 112 nanofluids with the presence of particle agglomerates. With this goal, the present paper 113 reports an experimental investigation of the rheological properties of CNT water-based 114 nanofluids stabilized by SDBS as surfactant. Here we focus our study on the influence of 115 particle concentration and temperature. In the first part, we present the CNT water-based 116 nanofluids used in the study. The experiments for rheological measurements are then detailed 117 in section 3. In section 4, experimental results are presented and discussed in terms of the 118 influence of particle content and temperature on the viscosity of CNT suspensions. We examine various theoretical viscosity models to predict the relative viscosity of CNT nanofluids at high shear rate and show that it is well represented by the modified Maron-

121 Pierce equation considering the influence of nanoparticles agglomerates.

122

\section{Materials}

\subsection{Nanofluid}

A CNT water-based suspension was provided by Nanocyl (Belgium). According to Nanocyl's specification, this suspension consists of MWCNTs (carbon purity 90\%) dispersed in a base fluid of de-ionized water and surfactant from ultrasonication, and was stable for several months. The dimensions of the nanotubes are $1.5 \mu \mathrm{m}$ in average length $\mathrm{L}$ and $9.2 \mathrm{~nm}$ in average diameter $\mathrm{d}$ respectively. This leads to an average aspect ratio $\mathrm{r}=\mathrm{L} / \mathrm{d} \approx 163$. The density of the nanotubes is $1800 \mathrm{~kg} / \mathrm{m}^{3}$. The weight fraction of nanotubes is $1 \%$. This corresponds to a volume fraction of $0.55 \%$. The surfactant used is sodium dodecyl benzene sulfonate (SDBS). The quantity of surfactant represents $2 \%$ of the total weight of the nanosuspension. As it is well known that carbon nanotubes have a hydrophobic surface, the surfactant is used to disperse and stabilize the particles and reduce the presence of aggregates [15]. The base fluid was also independently prepared and provided by Nanocyl.

\subsection{Suspensions preparation}

The suspension provided by Nanocyl was used as a starting material to prepare suspensions with various lower mass concentrations of $0.75,0.5,0.2,0.1,0.05$ and $0.01 \%$. This corresponds to a volume fraction of $0.418,0.278,0.111,0.055,0.027$ and $0.00555 \%$ respectively. De-ionized water was used to dilute and prepare these suspensions. The appropriate mass of water was precisely measured and then introduced in the initial 
145 suspension to reach the required mass (or volume) fraction in nanotubes. The mixture was 146 stirred with a mixer for 30min and then remained at rest. The 30min stirring of each 147 suspension was repeated 24hours later. Mechanical stirring is used to ensure uniform 148 dispersion of nanoparticles and prevent initial agglomeration of nanoparticles in the base 149 fluid. Thereafter, all the suspensions were stored in a container at ambient temperature. No 150 observable sedimentation was noticed before rheological measurements. Following the same 151 procedure, the initial base fluid was also diluted to obtain the base fluids corresponding to the 152 different nanofluids previously prepared. The volume fraction of both tested nanofluids and corresponding base fluids are reported in Table 1.

154

\section{3. Experiments}

\section{$156 \quad 3.1$ Characterization of nanofluids}

157 Scanning Electron Microscopy (SEM) characterization of the starting nanofluid suspension

158 was preliminary performed to investigate the dispersion state of the nanoparticles within the 159 base fluid, and evaluate the presence and the size of the aggregates [25]. As reported in Figure

1601 , it is shown that the nanotubes are entangled and the starting nanofluid suspension is mainly in the form of aggregates with a maximal aggregate size about 4 times higher than the average length of the nanotubes.

163

164

165

166

167

168

169

170

171

172

173

174

175

176

177

178

179

\subsection{Rheological measurements}

Rheological measurements of nanofluid samples were performed using a stress controlled rheometer (Malvern Kinexus Pro) in a cone and plate configuration under controlled temperature. The temperature was controlled using a Peltier temperature control device located below the lower plate. Thermal clovers were also used to ensure constant temperature within the sample gap. For all experiments, the cone diameter was $60 \mathrm{~mm}$ and the cone angle was $1^{\circ}$.

The working temperature was varied from $0^{\circ} \mathrm{C}$ to $40^{\circ} \mathrm{C}$ with an interval of $10^{\circ} \mathrm{C}$. The higher temperature was limited to $40^{\circ} \mathrm{C}$ because the surfactant addition in nanosuspensions limits applications at high temperatures [5,6]. It has been shown that above $60^{\circ} \mathrm{C}$ the bonding between surfactant and nanoparticles can be damaged [2,4]. The nanofluid will also lose its stability and sedimentation of nanoparticles will occur [3]. For example, SDBS was observed to fail at elevated temperatures in [1]. This was also observed from our experiments at $50^{\circ} \mathrm{C}$, as the measured shear viscosity, which varies from one stress to another, indicates the 
unstable structure under shear (not reported here). The limitation of high temperature was also

181 used to prevent water evaporation from the sample due to the duration of the rheological experiments.

183 Each tested volume sample was taken from its container with a syringe-type automatic pipette and transferred to the lower plate, taking care that no air bubbles were entrapped in the sample. Hence, the cone is displaced to achieve the required sample gap. The excess of sample was eventually removed. The sample was allowed to equilibrate for $5 \mathrm{~min}$ before starting the experiment. It should be mentioned that a new sample was used for each measurement and that both cone and plate were cleaned between each measurement. Moreover, no high mixing or sonification was applied to the nanofluids before taking it for rheological measurement. Therefore, no preshear was applied to the samples before testing them. As a consequence, it is considered that each sample has been subjected to the same shear history before being tested. Actually, rheological properties of CNT nanofluids are sensitive to preshear history effect [25].

194 Stress-controlled measurements were performed by imposing a logarithmic stress ramp under steady-state conditions with maximum step duration of 180s. Once a steady-state flow was achieved and maintained for $10 \mathrm{~s}$, the shear rate was measured. The applicability of the shear stress range was subject to a preliminary evaluation to ensure steady-state flow at low shear stress, and to avoid flow instability and sample ejection at high shear stress, in particular for suspensions with lower mass content in particles. The end value of the shear stress ramp may vary following the tested suspension, and was set in order to reach a shear rate of $1000 \mathrm{~s}^{-1}$ for each nanosuspension. The experiments were also repeated at least once to both verify the repeatability of the shear viscosity measurement and the suspension stability with time. Following the same experimental procedure, another series of experiments were conducted to evaluate the rheological behavior of the base fluids corresponding to the nanofluids with different volume fraction. The torque resolution of the rheometer is $0.1 \mathrm{nNm}$. This means that the uncertainty of shear stress with the cone and plate geometry used is $1.710^{-3} \mathrm{~Pa}$. The angular velocity resolution is at least $10 \mathrm{nrad} / \mathrm{s}$. The uncertainty of shear rate, which only depends on angular velocity and cone angle, is less than $5.7310^{-7} \mathrm{~s}^{-1}$. This leads to an uncertainty in apparent viscosity less than $4 \%$ within the shear rate range investigated.

\section{Results and discussion}

\subsection{Viscosity measurement validation and viscosity of base fluids}


214 To evaluate the rheometer uncertainty and the experimental procedure, distilled water was

215 tested at $20^{\circ} \mathrm{C}$ following the procedure described here before and for two replicates. As 216 expected, distilled water exhibits a Newtonian behavior within the shear rate range 217 investigated. The viscosity value of distilled water was 1.0345 , which closely matches with its 218 theoretical values at $20^{\circ} \mathrm{C}$. The relative deviation is less than $3.5 \%$. This is of the same order 219 of magnitude as the experimental uncertainty.

220 Figure 2 reports the shear viscosity of the base fluid for the starting nanofluid at $0.55 \%$ in 221 volume concentration of nanoparticles. It is observed from figure 2 that this base fluid 222 behaves in Newtonian manner, as the apparent viscosity is constant within the shear rate range investigated. The shear viscosity value of this base fluid at $20^{\circ} \mathrm{C}$ is $1.129 \mathrm{mPa}$.s. This is higher than the viscosity of de-ionized water, showing the influence of SDBS. It is also shown from figure 1 that the viscosity of the base fluid decreases when the temperature is increased. The shear viscosity of the base fluid of the nanofluid at $0.0055 \%$ in volume fraction of nanoparticles is reported in figure 3. We observe than this base fluid is also Newtonian as the shear viscosity is constant within the shear rate range investigated and its viscosity is very close to the one of de-ionized water. A similar effect of temperature increase is also obtained for the viscosity of this base fluid. Too, a Newtonian behavior for all tested base fluids is thus observed.

232 In Table 1, the shear viscosities of all base fluids (corresponding to all tested nanofluids) are given as a function of volume fraction in SDBS. Table 1 shows that, for all the tested temperatures, the shear viscosity of base fluids slowly decreases with the decrease of SDBS volume fraction. This can be explained by the dilution of the base fluids and the reduction of the presence of SDBS. When the volume fraction of SDBS is lower than $0.169 \%$, the shear viscosity of the base fluid is quite constant and tends to the viscosity of water. As reported before, when the temperature increases, the shear viscosity of the base fluids decreases.

\subsection{Viscosity of nanofluids: Influence of concentration and temperature}

241 Figure 4 reports the evolution of the shear viscosity depending on shear rate for CNT 242 nanofluid at $0.278 \%$ in volume fraction and for all the tested temperatures. Figure 4 shows 243 that the nanofluid behaves as a shear-thinning fluid as the shear viscosity decreases when the 244 shear rate increases. The good agreement between two replicates of rheological measurement 245 for this nanofluid at $10^{\circ} \mathrm{C}$ can also be noted in figure 4 . This shows the repeatability of the 246 measurement and the stability of the nanofluid. Similar trends were obtained for all nanofluids 247 and temperatures. 
In figure 5, the shear viscosity of CNT nanofluid at $0.0055 \%$ in volume fraction is plotted against shear rate. Comparison of figure 4 and 5 shows that the rheological behavior of nanofluids is strongly dependent on the volume fraction of nanotubes within the nanofluids. This trend can also be shown by figure 6, when we compare the shear viscosity of all nanofluids at $20^{\circ} \mathrm{C}$. In figure 6 we observe that the higher the concentration, the higher the shear-thinning behavior and the extent of the shear-thinning region. The transition between shear-thinning behavior and Newtonian behavior of the nanofluids is for a volume fraction of $0.055 \%$. Figure 5 also shows that the shear viscosity increases with CNT concentration for a given shear rate. It should be noted that the nanofluid with lower concentration have lower shear viscosity that of the base fluid. This effect is probably due to the lubricative effect of nanoparticles [26].

It is shown from figures 4 and 5 that temperature has a strong effect on the rheological properties of CNT nanofluids. Actually, the nanofluids viscosity decreases with increasing temperature, as generally reported for a wide class of nanofluids and previously observed for the base fluids.

As reported in the literature, the shear-thinning effect, in addition to the effect of nanotubes length, can be attributed to the disagglomeration of the nanotube clusters and the alignment of the agglomerates and nanotubes during shear, resulting in less viscous force. It can be noted that the shear-thinning behavior of the studied CNT nanofluids is stronger than the one reported by Kanagaraj et al. [22], which indicates a great effect of the aspect ratio and the agglomerates network of the nanotubes on the shear viscosity. In the present work, the aspect ratio of the nanotubes is higher than the aspect ratio $(r=L / d \approx 8)$ of the nanoparticles used in [22]. Our results are also consistent with the work of Ding et al. [27], which showed the shear-thinning behavior of aqueous suspension of carbon nanotubes at 20 and $40^{\circ} \mathrm{C}$ within the shear rate range 1 to $1000 \mathrm{~s}^{-1}$. Shear-thinning of MWCNT-based aqueous nanofluids was also observed by Garg et al. [20] at 15 and $30^{\circ} \mathrm{C}$ for low shear rate range between 0 and $80 \mathrm{~s}^{-1}$ and by Maré et al. [28] for temperatures ranging from $0^{\circ} \mathrm{C}$ to $10^{\circ} \mathrm{C}$.

275 Figure 7 reports the relative viscosity at a high shear rate of $1000 \mathrm{~s}^{-1}$, defined as the ratio of the 276 CNT nanofluid viscosity at high shear rate to the viscosity of the base fluid at the same shear rate, as a function of all tested temperatures. While nanofluids and base fluids are strongly dependent on temperature, it is also observed from figure 7 that the relative viscosity is quite constant for the tested temperatures under the experimental uncertainty. The relative viscosity of nanofluids at a high shear rate in function of nanofluid volume fraction is plotted in Figure 
mainly evidenced for nanoparticles volume fraction of $0.055 \%$ and that the viscosity of nanofluid is 6 times higher than the viscosity of the base fluid for nanoparticles volume fraction of $0.55 \%$. The influence of volume fraction on the enhancement of relative viscosity of nanofluids is investigated in the following section, considering first the shape of the nanotubes and then the presence of agglomerates network.

\subsection{Viscosity models: prediction and comparison with experimental data}

Some theoretical formulas have been developed to relate the relative viscosity of nonaggregating colloidal suspensions or nanofluids to particle volume fraction. They are derived from the pioneering model of Einstein [29]. This model is based on the assumption of viscous fluid containing non-interacting hard spheres under particle volume fraction less than $1 \%$.

$$
\mu_{r}=(1+2.5 \phi)
$$

where $\mu_{\mathrm{r}}$ is the relative viscosity as defined by the ratio of the viscosity of the nanofluid $\mu_{\mathrm{n}}$ to the viscosity of the base fluid $\mu_{\mathrm{bf}}$, and $\phi$ is the volume fraction of nanoparticle in base fluid. Later, Einstein's equation was extended by Brinkman [30] to suspensions with moderate particle volume fraction, typically less than $4 \%$.

$$
\mu_{r}=\frac{1}{(1-\phi)^{2.5}}
$$

Considering the Brownian motion of nanoparticles and the interaction between a pair of particles, Batchelor [31] proposed the following equation.

$$
\mu_{r}=\left(1+\eta \phi+k_{H} \phi^{2}+\ldots\right)
$$

In equation (3), $\eta$ is the intrinsic viscosity and $k_{H}$ is the Huggins' coefficient. The values of $\eta$ and $\mathrm{k}_{\mathrm{H}}$ are 2.5 and 6.5 , respectively, for spherical particles.

For higher particle volume fraction, the Krieger-Dougherty relationship is considered as an efficient model to relate the viscosity of non-aggregating colloidal suspensions or nanofluids to particle volume fraction. This relationship is written as follows [32]: 


$$
\mu_{r}=\left(1-\frac{\phi}{\phi_{m}}\right)^{-\eta \phi_{m}}
$$

where $\eta$ is the Einstein coefficient, $\eta=2.5$, and $\phi_{\mathrm{m}}$ is the particle volume fraction when the viscosity is infinite, defined as the maximum volume fraction. Typically, $\phi_{\mathrm{m}} \approx 0.65$ for random packing of hard spheres.

An equation with the same functional form was derived by Maron and Pierce from consideration of the Ree-Eyring flow equations [33,34]. Noting that equation 5 was obtained from a minimum principle applied to the energy dissipated by viscous effects.

$$
\mu_{r}=\left(1-\frac{\phi}{\phi_{m}}\right)^{-2}
$$

The previous two equations reduce to the Einstein and Batchelor equations at first and second order, respectively. The Maron-Pierce equation can also be used to predict the relative viscosity of fiber suspensions, the maximum volume fraction $\phi_{\mathrm{m}}$ depending also on the aspect ratio of the fibers [35]. So, the value of the maximum volume fraction decreases with increasing aspect ratio. A value of $\phi_{\mathrm{m}} \approx 0.0361$ is here obtained due to the aspect ratio of the nanotubes used [35]. This is very low in comparison of the maximum volume fraction of spheres, thus showing the effect of nanoparticle shape. As mentioned before, many nanoparticles have a non-spherical shape. So Brenner and Condiff [36] have also developed a viscosity model to consider the shape effects in dilute suspension. So, for rod-like particles, the Brenner-Condiff equation is applicable for a volume fraction up to $1 / \mathrm{r}^{2}$ (this corresponds here to $0.004 \%$ in volume) and for viscosity at high shear rate, where $r$ is the aspect ratio of nanoparticles.

$$
\mu_{r}=(1+\eta \phi)
$$

with

$$
\eta=\frac{0.312 r}{\ln 2 r-1.5}+2-\frac{0.5}{\ln 2 r-1.5}-\frac{1.872}{r}
$$


346 In the presence of agglomerates, it was reported that the relative viscosity of aggregating 347 suspensions or nanofluids can be modelled from the application of the fractal concept [37]. 348 So, the geometry of the aggregates can be described as a fractal like structure with a fractal index D. Assuming that the aggregates density change with the radial position and is not uniform in the nanofluid [8], the effective volume fraction of nanoparticles, denoted $\phi_{\mathrm{a}}$, is written as follows

$$
\phi_{a}=\phi\left(\frac{a_{a}}{a}\right)^{3-D}
$$

354

355 356

where $a_{a}$ and a are the aggregates and primary nanoparticles radii respectively.

This leads to the modified Krieger-Dougherty formula given by equation (9) which was successfully applied to model the viscosity enhancement of aggregating nanofluids made of spherical particles $[11,38]$ and rod-like particles $[8,9,39]$.

$$
\mu_{r}=\left(1-\frac{\phi_{a}}{\phi_{m}}\right)^{-\eta \phi_{m}}
$$

As a consequence, the modified Maron-Pierce equation can also be written [40]:

$$
\mu_{r}=\left(1-\frac{\phi_{a}}{\phi_{m}}\right)^{-2}
$$

As previously reported [8], the fractal index D can depend on the type of aggregation, particle size and shape and shear flow condition. So, for aggregating nanofluids with spherical particles, D has been reported to be between 1.6 and 2.3 [41-43]. However, a value of 1.8 is typically used [40,44]. For aggregating nanofluids with nanorods or nanotubes, D varies between 1.5 and 2.45 as reported by [39]. Mohraz et al. [45] showed that the fractal index depends on the aspect ratio of nanorods. They reported that $\mathrm{D}$ increases from 1.8 to 2.3 for $\mathrm{r}$ ranging from 1 to 30.6 respectively. A value of 2.1 is generally taken $[8,45,46]$. Such a value was also obtained by Chatterjee and Krishnamoorti [47] for single walled carbon nanotubes (SWCNTs) dispersed in PEO, and by Khalkhal and Carreau [48] for MWCNTs dispersed in 
epoxy resin, and a value of 2.05 was reported by Chen et al. [49] for SWCNTs dispersed in aqueous supensions. Based on the above, a value of 2.1 was taken for the fractal index in the present work.

379 Comparison of the experimental data with the predicted data from the above formulas is shown in figure 8. It is clear that the Einstein, Brinkman, Brenner and Condiff and MaronPierce formulas cannot predict the relative viscosities of the CNT water based nanofluids for volume concentration exceeding $0.027 \%$. In addition, the difference between the experimental and the computed values increases with the volume fraction. This result shows the strong influence of agglomerates which significantly increase the effective volume of the nanotubes and thus the relative viscosity of the nanofluid. This also confirms the conclusions reported previously from the steady state apparent viscosity curves.

Nevertheless, figure 8 shows excellent agreement between the prediction of the modified model of Maron-Pierce and experimental data when $\mathrm{a}_{\mathrm{a}} / \mathrm{a} \approx 4.41$, as the average deviation of experimental relative viscosity compared to the model is less than $5 \%$. If a is taken as the average lenght of the nanotubes, this leads to a maximum aggregates size close to $6.6 \mathrm{~nm}$, which is in quite good agreement with the maximum size of CNT aggregates determined from SEM analyses [25]. A comparison of our results with the data of Chen et al. [38] (EGspherical $\mathrm{TiO}_{2}, \mathrm{~d}=25 \mathrm{~nm} \mathrm{a} / \mathrm{a}=3.34, \mathrm{D}=1.8, \phi_{\mathrm{m}}=0.605$ ), the data of Kole and Dey [11] (gear oilspherical $\left.\mathrm{CuO}, \mathrm{d}=40 \mathrm{~nm}, \mathrm{a}_{\mathrm{a}} / \mathrm{a}=7.15, \mathrm{D}=1.7, \phi_{\mathrm{m}}=0.605\right)$ and the data of Chen et al. [8] (EGTNT, $\left.r \approx 10, a_{a} / a=9.46, D=2.1, \phi_{m}=0.3\right)$, suggests that the size and the aspect ratio are an important factor in the formation of nanoclusters within the nanofluids in spite of the use of surfactant. This shows the influence of particle length on the entanglement of the nanoparticles, the presence and the size of agglomerates.

\section{Conclusion}

401 We have presented an experimental investigation of the rheological properties of water-based nanofluids containing carbon nanotubes (CNT) with large aspect ratio. The influence of particle concentration and temperature on the viscosity of the nanofluids was discussed and the nanofluids were shown to behave as a shear-thinning material at high particle content. For lower particle content, the nanofluids behave in Newtonian manner. It was also reported that temperature affects the viscosity of nanofluids and base fluids but that the relative viscosity of nanofluids at high shear rate is independent of temperature. The relative viscosity of 
presence of aggregates, and can be modelled by the Maron-Pierce equation considering the

410 influence of nanoparticles agglomerates. An average maximum size of aggregates was thus

411 evaluated and favourably compared with SEM characterization previously done. The results

412 of this experimental study also show the relevance of the rheological characterization

413 concerning the presence and the structural information of nanofluids aggregates and can

414 contribute to the understanding of the thermal properties of such materials.

\section{Acknowledgments}

Nanocycl Belgium is gratefully acknowledged for providing the CNT water based nanofluid.

\section{References}

[1] D.S. Wen, Y.L. Ding, Effective thermal conductivity of aqueous suspensions of carbon nanotubes (nanofluids), J. Thermophys. Heat Transfer 18 (2004) 481-485.

[2] M.J. Assael, I.N. Mataxa, J. Arvanitidis, D. Christophilos, C. Lioutas, Thermal conductivity enhancement in aqueous suspensions of carbon multi-walled and double-walled nanotubes in the presence of two different dispersants, Int. J. Thermophys. 26 (2005) 647664.

[3] X.Q. Wang, A.S. Mujumdar, Heat transfer characteristics of nanofluids: A review, Int. J. Therm. Sci. 46 (2007) 1-19.

[4] S.M.S. Murshed, K.C. Leong, C. Yang, Investigations of thermal conductivity and viscosity of nanofluids, Int. J. Therm. Sci. 47 (2008) 560-568.

[5] D. Wen, S. Lin, S. Vafaei, K. Zhang, Review of nanofluids for heat transfer applications, Particuology 7 (2009) 141-150.

[6] D. Wu, H. Zhu, L. Wang, L. Liua, Critical issues in nanofluids preparation, characterization and thermal conductivity, Curr. Nanosci. 5 (2009) 103-112.

[7] H. Chen, W. Yang, Y. He, Y. Ding, L. Zhang, C. Tan, A.A. Lapkin, D.V.Bavykin, Heat transfer and flow behaviour of aqueous suspensions of titanate nanotubes (nanofluids), Powder Tech 183 (2008) 63-12.

[8] H. Chen, Y. Ding, A.A. Lapkin, X. Fan, Rheological behaviour of ethylene glycol-titanate nanotube nanofluid, J. Nanopart. Res. 11 (2009) 1513-1520.

[9] H. Chen, Y. Ding, A.A. Lapkin, Rheological behaviour of nanofluids containing tube/rodlike nanoparticles, Powder Tech. 194 (2009) 132-141. 
[10] S.Q. Zhou, R. Ni, D. Funfschilling, Effects of shear rate and temperature on viscosity of alumina polyaphaolefins nanofluids, J. Appl. Phys. 107 (2010) 054317.

[11] M. Kole, T.K. Dey, Effect of aggregation on the viscosity of copper oxide-gear oil nanofluids, Int. J. Thermal Sci. 50 (2011) 1741-1747.

[12] P.J. Harris, Carbon nanotubes and related structures: new materials for the $21^{\text {st }}$ century, Cambridge, University Press, 1999.

[13] Y. Otsubo, M. Fujiwara, M. Kouno, K. Edamura, Shear-thickening flow of suspensions of carbon nanofibers in aqueous PVA solutions, Rheol. Acta 46 (2007) 905-912.

[14] M.F.Yu, B.S.Files, S. Arepalli, R.S. Ruoff, Tensile loading of ropes of single wall carbon nanotubes and their mechanical properties, Physical Rev Lett 84 (2000) 5552-5.

[15] L. Vaisman, H.D.Wagner, G. Marom, The role of surfactants in dispersion of carbon nanotubes, Adv. Coll. Int. Sci. 128-130 (2006) 37-46.

[16] L. Chen, H. Xie, W. Yu, Nanofluids containing carbon nanotubes treated by mechanochemical reaction, Thermoch. Acta 477 (2008) 21-24.

[17] H. Wang, Dispersing carbon nanotubes using surfactants, Curr. Opinion Coll. Interface Sci. 14 (2009) 364-371.

[18] A. Nasiri, M. Shariaty-Niasar, A. Rashidi, A. Amrollahi, R. Khodafarin, Effect of dispersion method on thermal conductivity and stability of nanofluid, Exp. Thermal Fluid Sci. 35 (2011) 717-723.

[19] T.X. Phuoc, M. Massoudi, R.H. Chen, Viscosity and thermal conductivity of nanofluids containing carbon nanotubes stabilized by chitosan, Int. J. Thermal Sci. 50 (2011) 12-18.

[20] P. Garg, L.A. Jorge, C. Marsh, T.A. Carlson, D.A. Kessler, K. Annamalai, An experimental study on the effect of ultrasonication on viscosity and heat transfer performance of multi-wall carbon nanotube-based aqueous nanofluids, Int. J. Heat Mass Transfer 52 (2009) 5090-5101.

[21] Y. Yang, E.A. Grulke, Z.G. Zhanh, G. Wu, Thermal and rheological properties of carbon nanotube-in-oil dispersions, J. Appl. Phys. 99 (2006) 114307.

[22] S. Kanagaraj, F.R Varabda, A. Fonseca, J. Ponmozhi, J.A. Lopez da Silva, M.S.A. Oliveira et al, Rheological study of nanofluids at different concentration of carbon nanotubes, 19th National \& 8th ISHMT-ASME Heat Mass Transfer Conf., January 3-5, 2008 hvderabad, India (paper NFF-7).

[23] J. Ponmozhi, F.A.M.M. Gonçalves, A.G.M. Feirrera, I.M.A Fonseca , S. Kanagaraj, M. Martins, M.S.A. Oliveira, Thermodynamic and transport properties of CNT water based nanofluids, J. Nano Res. 11 (2010) 101-106. 
[24] B. Aladag, S. Halelfadl, N. Doner, T. Maré, S. Duret, P. Estellé, Experimental investigations of the viscosity of nanofluids at low temperatures, App. Energy, 97 (2012) 876-880.

[25] P. Estellé, S. Halelfadl, N. Doner, T. Maré, Shear flow history effect on the viscosity of carbon nanotubes water based nanofluid, Current Nanoscience, 9/2 (2013) 225-230.

[26] L. Chen, H. Xie, W. Yu, Y. Li, The rheological behaviors of nanofluids containing multiwalled carbon nanotube, J. Disp. Sci. Tech. 32 (2011) 550-554.

[27] Y. Ding, H. Alias, D. Wen, R.A. Williams, Heat transfer of aqueous suspensions of carbon nanotubes (CNT nanofluids), Int. J. Heat Mass Transfer 49 (2006) 240-250.

[28] T. Maré, , S. Halelfadl, O. Sow, P. Estellé, S. Duret, F. Bazantay, Comparison of the thermal performances of two nanofluids at low temperature in a plate heat exchanger, Exp. Thermal Fluid Sci 35 (2011) 1535-1543.

[29] A. Einstein, Investigations on the Theory of the Brownian Movement, Dover Publications, Inc., New York, 1956.

[30] H.C. Brinkman, The viscosity of concentrated suspensions and solutions, J. Chem. Phys. 20 (1952) 571-581.

[31] G. Batchelor, The effect of Brownian motion on the bulk stress in a suspension of spherical particles, J. Fluid Mech. 83 (1977) 97-117.

[32] I.M. Krieger, T.J. Dougherty, A mechanism for non-Newtonian flow in suspension of rigid spheres, J. Trans. Soc. Rheol. 3 (1959) 137-152.

[33] S.H. Maron, P.E. Pierce, Application of Ree-Eyring generalized flow theory to suspensions of spherical particles, J. Colloid Sci. 11 (1956) 80-95.

[34] M.M. Cross, Viscosity-concentration-shear rate relations for suspensions, Rheol. Acta 14 (1975) 402-403.

[35] S. Mueller, E.W. Llewellin, H.M. Mader, The rheology of suspensions of solid particles. Proc. of The Royal Society A, 466 (2010) 1201-1228.

[36] H. Brenner, D.W. Condiff, Transport mechanics in systems of orientable particles. Part IV. Convective transport, J. Colloid Int. Sci. 47 (1974) 199-264.

[37] W. Wolthers, M.H.G. Duits, D. van den Ende, J. Mellema, Shear history dependence of aggregated colloidal dispersions, J. Rheol. 40 (1996) 799-811.

[38] H. Chen, Y. Ding, C. Tan, Rheological behavior of nanofluids, New J. Phys. 9 (2007) 367.

[39] H. Chen, S. Witharana, Y. Jin, C. Kim, Y. Ding, Predicting thermal conductivity of liquids suspensions of nanoparticles (nanofluids) based on rheology, Particuology 7 (2009) 151-157. 
552 [40] J. Chevalier, O. Tillement, F. Ayela, Structure and rheology of $\mathrm{SiO}_{2}$ nanoparticle suspensions under very high shear rates, Phys Rev E 80 (2009) 051403.

[41] T.D. Waite, J.K. Cleaver, J.K. Beattie, Aggregation kinetics and fractal structure of gamma-alumina assemblages, J. Colloid Int. Sci. 241 (2001) 333-339.

[42] B.X. Wang, L.P. Zhou, X.F. Peng, A fractal model for predicting the effective thermal conductivity of liquid with suspension of nanoparticles, Int. J. Heat Mass Transf. 46 (2003) 2665-2672.

[43] Y. Xuan, Q. Li, W. Hu, Aggregation structure and thermal conductivity of nanofluids, AIChE J. 49 (2003) 1038-1043.

[44] R. Prasher, P.E. Phelan, P. Bhattacharya, Effect of aggregation kinetics on the thermal conductivity of nanoscale colloidal solutions (nanofluid), Nano Lett. 6 (2006) 1529.

[45] A. Mohraz, D.B. Moler, R.M. Ziff, M.J. Solomon, Effect of monomer geometry on the fractal structure of colloidal rod aggregates, Phys. Rev. Lett. 92 (2004) 155503.

[46] J.M. Lin, T.L. Lin, U. Jeng, Y. Zhong, C. Yeh, T. Chen, Fractal aggregates of the Pt nanoparticles synthesized by the polyol process and poly(N-vinyl-2-pyrrolidone) reduction, J. Appl. Crystallogr. 40 (2007) 540-543.

[47] T. Chatterjee, R. Krishnamoorti, Dynamic consequences of the fractal network of nanotube-poly(ethylene oxide) nanocomposites, Phys. Rev. E Stat. Nonlinear Soft Matter Phys. 75 (2007) 050403.

[48] F. Khalkhal, P.J. Carreau, Scaling behavior of the elastic properties of non-dilute MWCNT-epoxy resine, Rheol. Acta 50 (2011) 717-728.

[49] Q. Chen, C. Saltiel, S. Manickavasagam, L.S. Schadler, R.W. Siegel, H. Yang , Aggregation behavior of single-walled carbon nanotubes in dilute aqueous suspension, J. 585 
Figure Captions

Figure 1. SEM image taken from dried starting nanofluid [25] (This figure was reprinted with the permission of Bentham Science Publishers)

Figure 2. Apparent shear viscosity of the base fluid corresponding to the nanofluid with $0.55 \%$ in CNT volume fraction - Influence of temperature.

Figure 3. Apparent shear viscosity of the base fluid corresponding to the nanofluid with $0.0055 \%$ in CNT volume fraction- Influence of temperature.

Figure 4. Apparent shear viscosity of nanofluid with $0.278 \%$ in CNT volume fraction Influence of temperature.

Figure 5. Apparent shear viscosity of nanofluid with $0.0055 \%$ in CNT volume fraction Influence of temperature.

Figure 6. Viscosity of nanofluids at $20^{\circ} \mathrm{C}$ as a function of shear rate for different volume fraction of nanotubes.

Figure 7. Relative viscosity of nanofluids as a function of particle volume content and temperature.

Figure 8. Relative viscosity of nanofluids as a function of particle volume content and temperature - Comparison of experimental data and viscosity models.

\section{Table Caption}

Table 1. Volume fraction of tested nanofluids and corresponding base fluids, and shear viscosity of base fluids as a function of SDBS volume fraction and temperatures. 
623

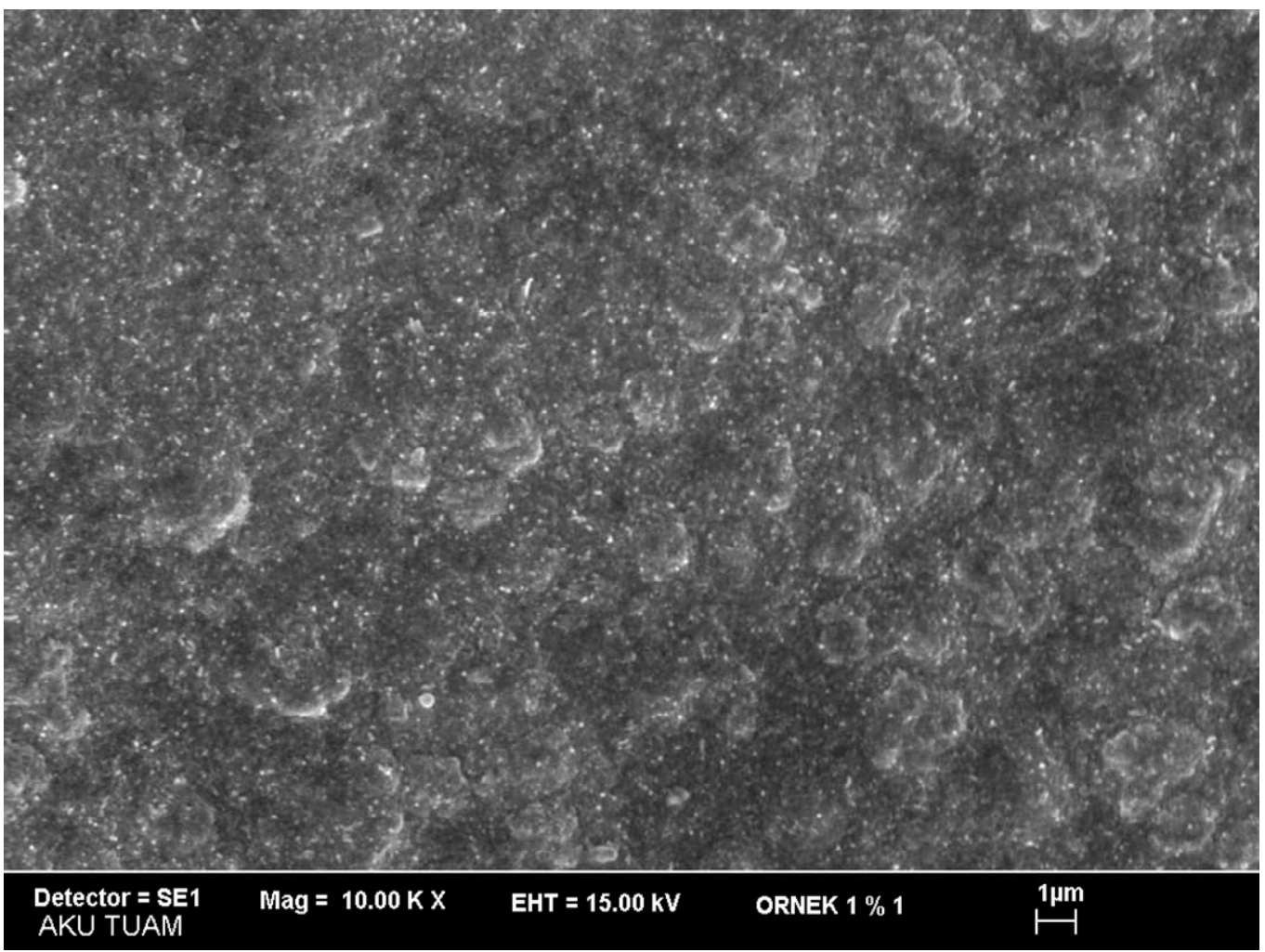

Figure 1. SEM image taken from dried starting nanofluid [25] (This figure was reprinted with the permission of Bentham Science Publishers)

624

625 


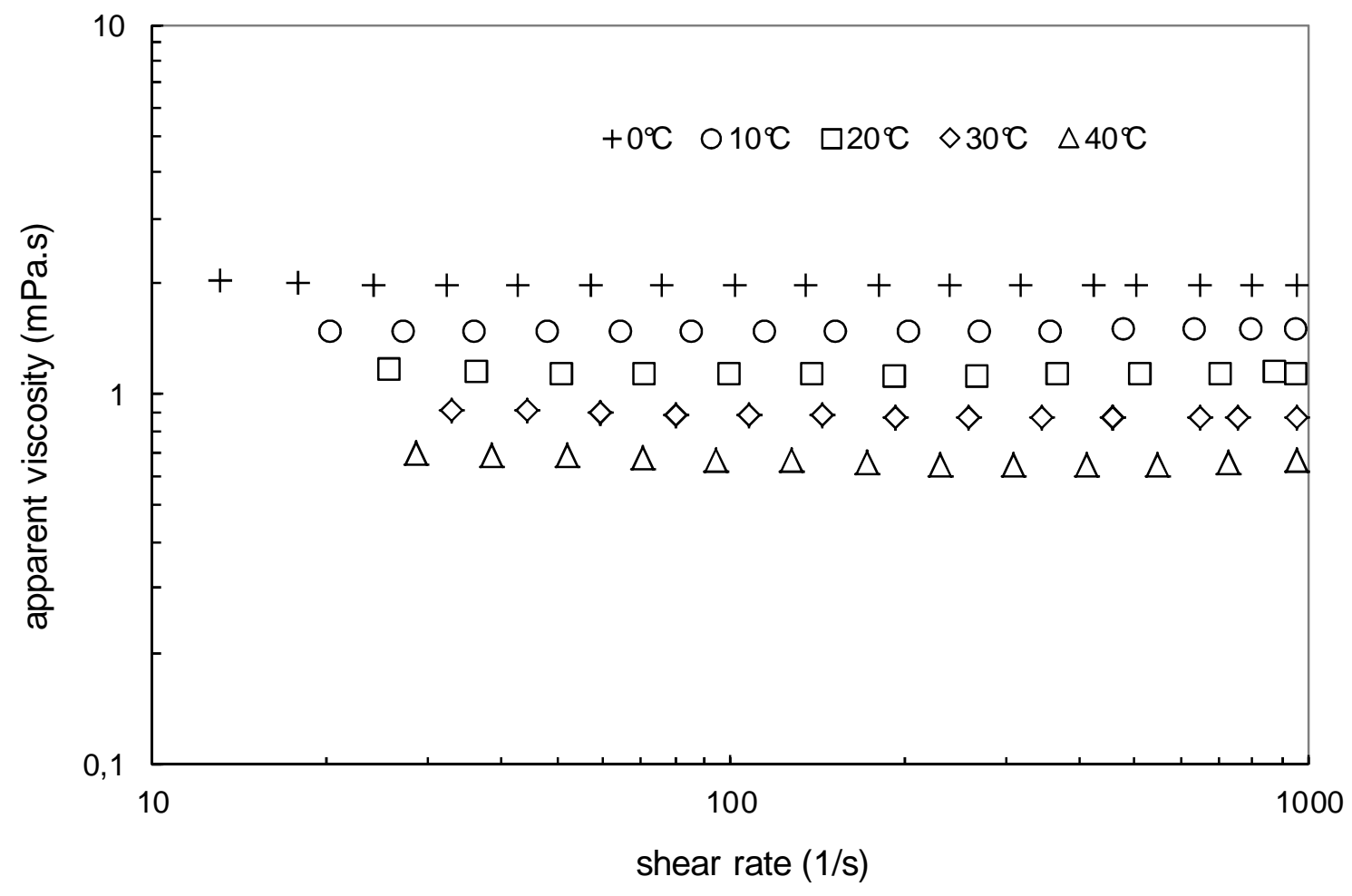

627

628 Figure 2. Apparent shear viscosity of the base fluid corresponding to the nanofluid with 629 $0.55 \%$ in volume fraction - Influence of temperature.

630 
631

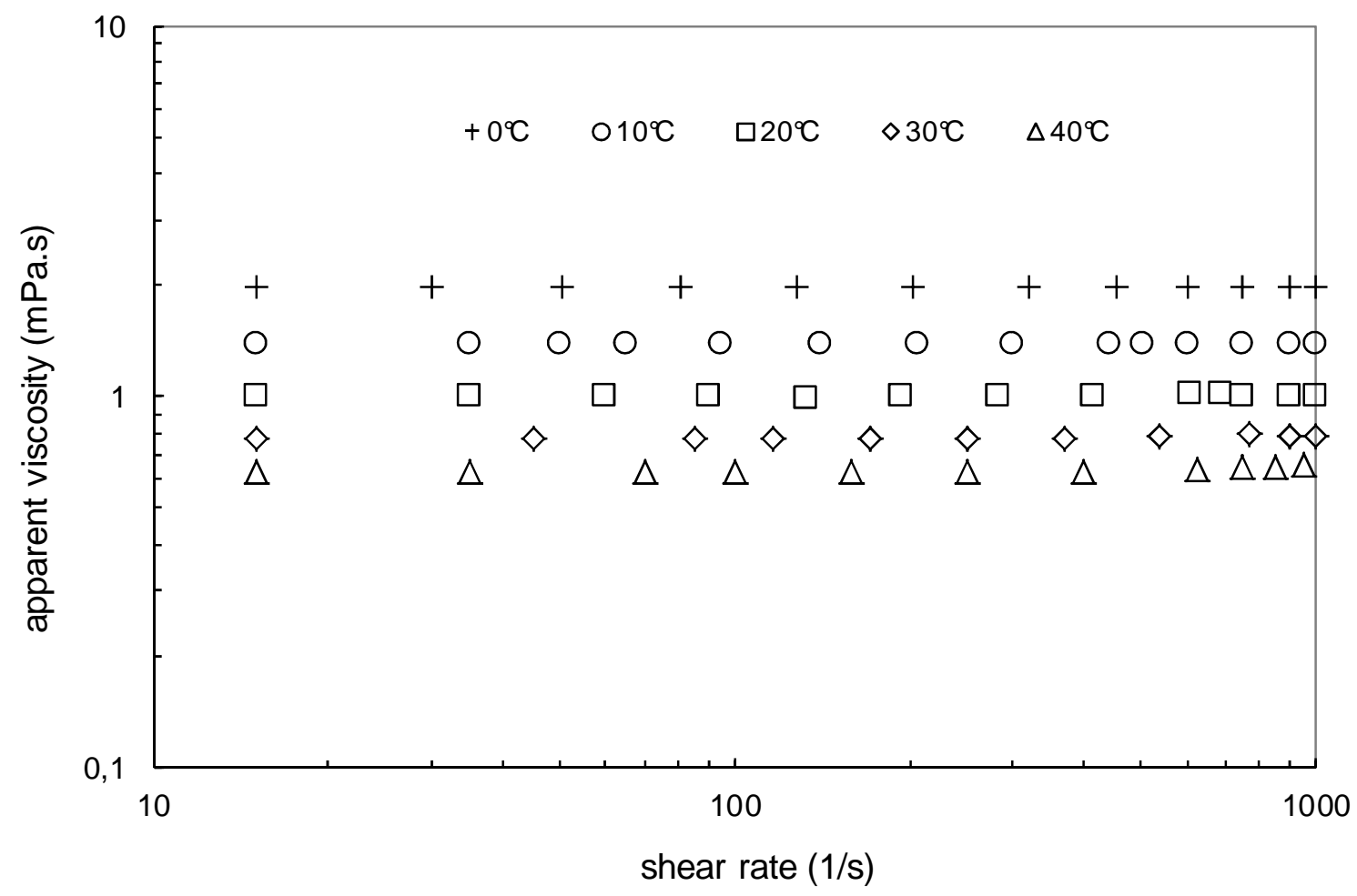

632

633 634 635

636 


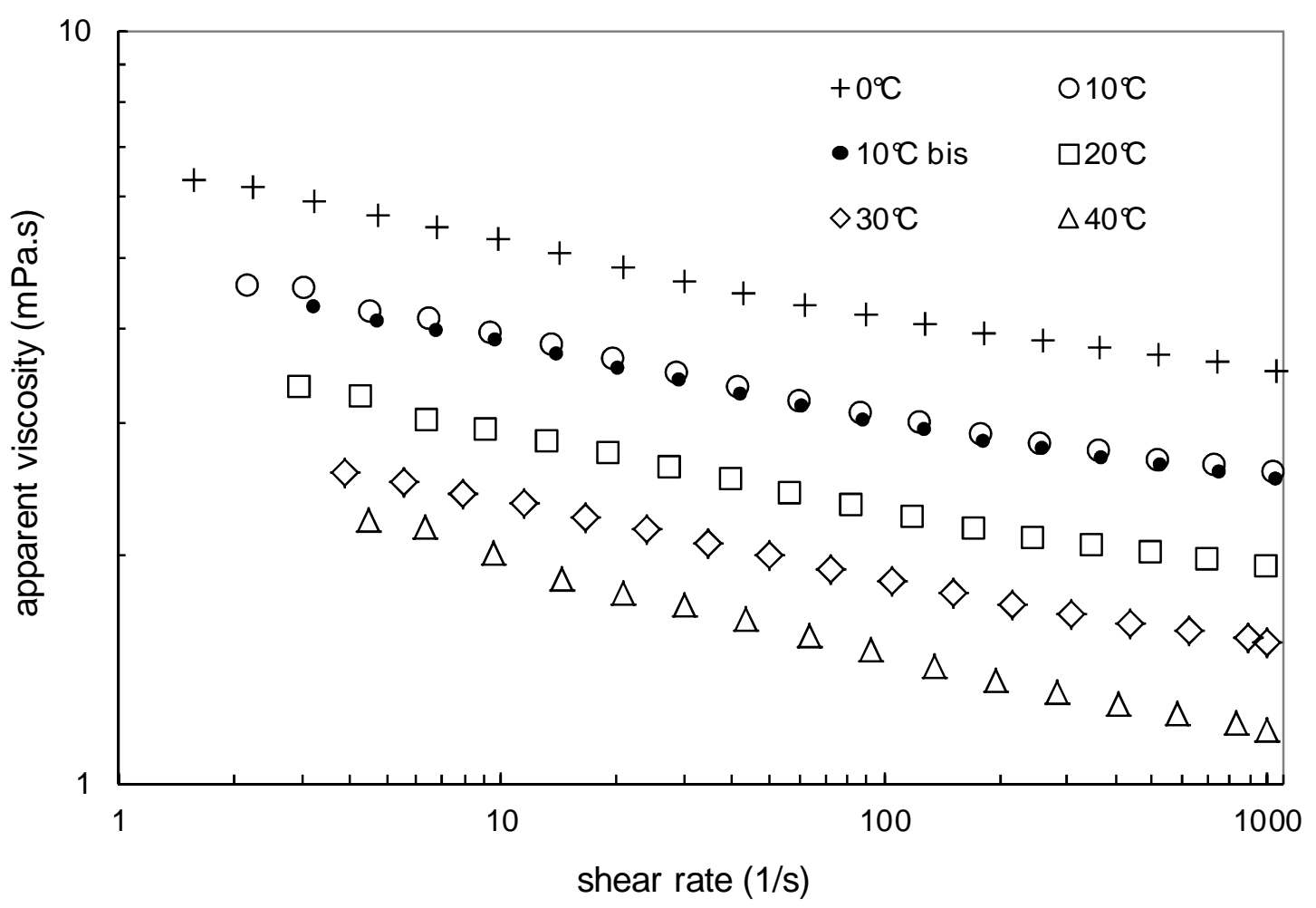

Figure 4. Apparent shear viscosity of nanofluid with $0.278 \%$ in CNT volume fraction 641 


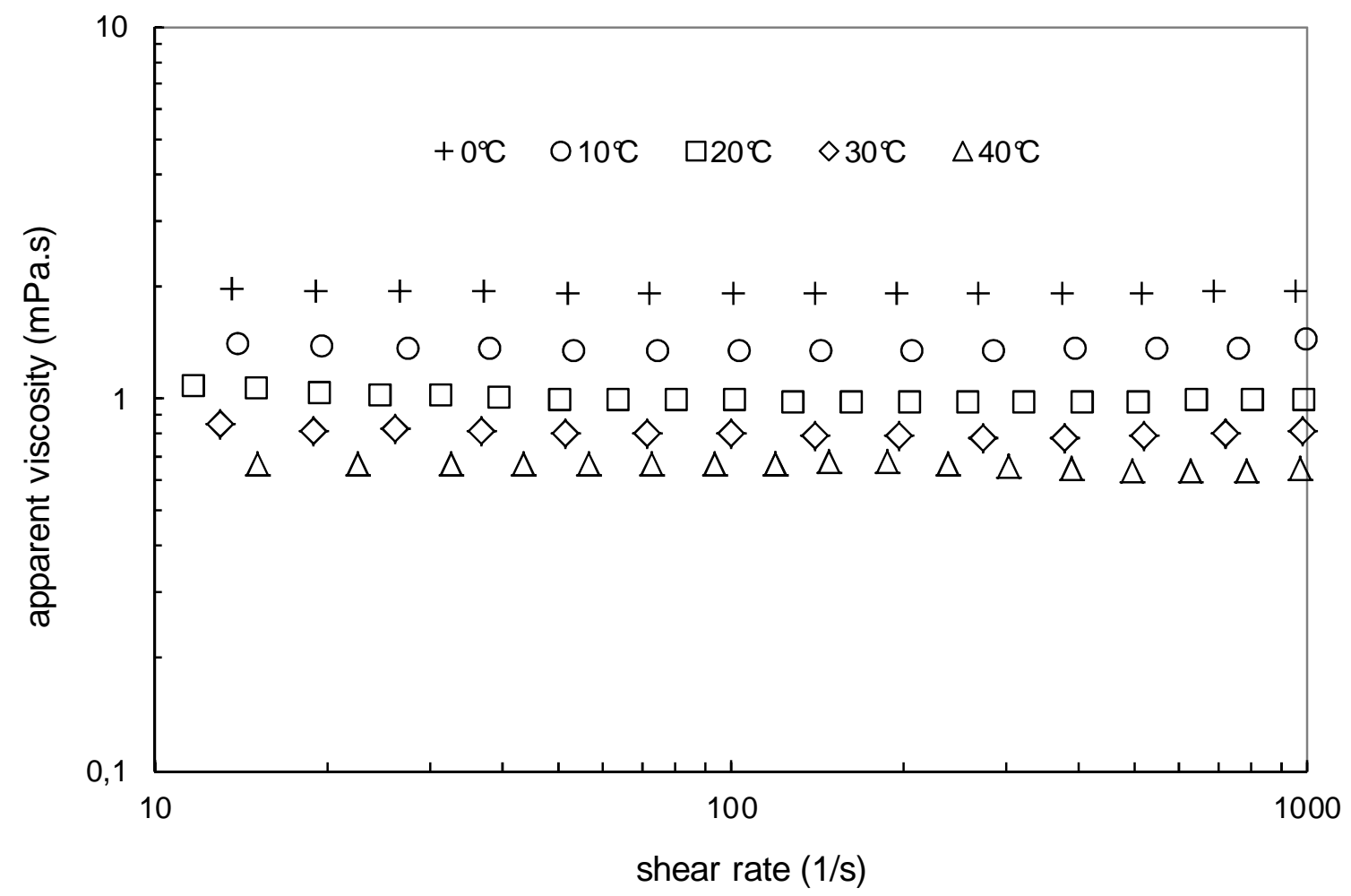

643 644 645 646
Figure 5. Apparent shear viscosity of nanofluid with $0.0055 \%$ in CNT volume fraction Influence of temperature. 


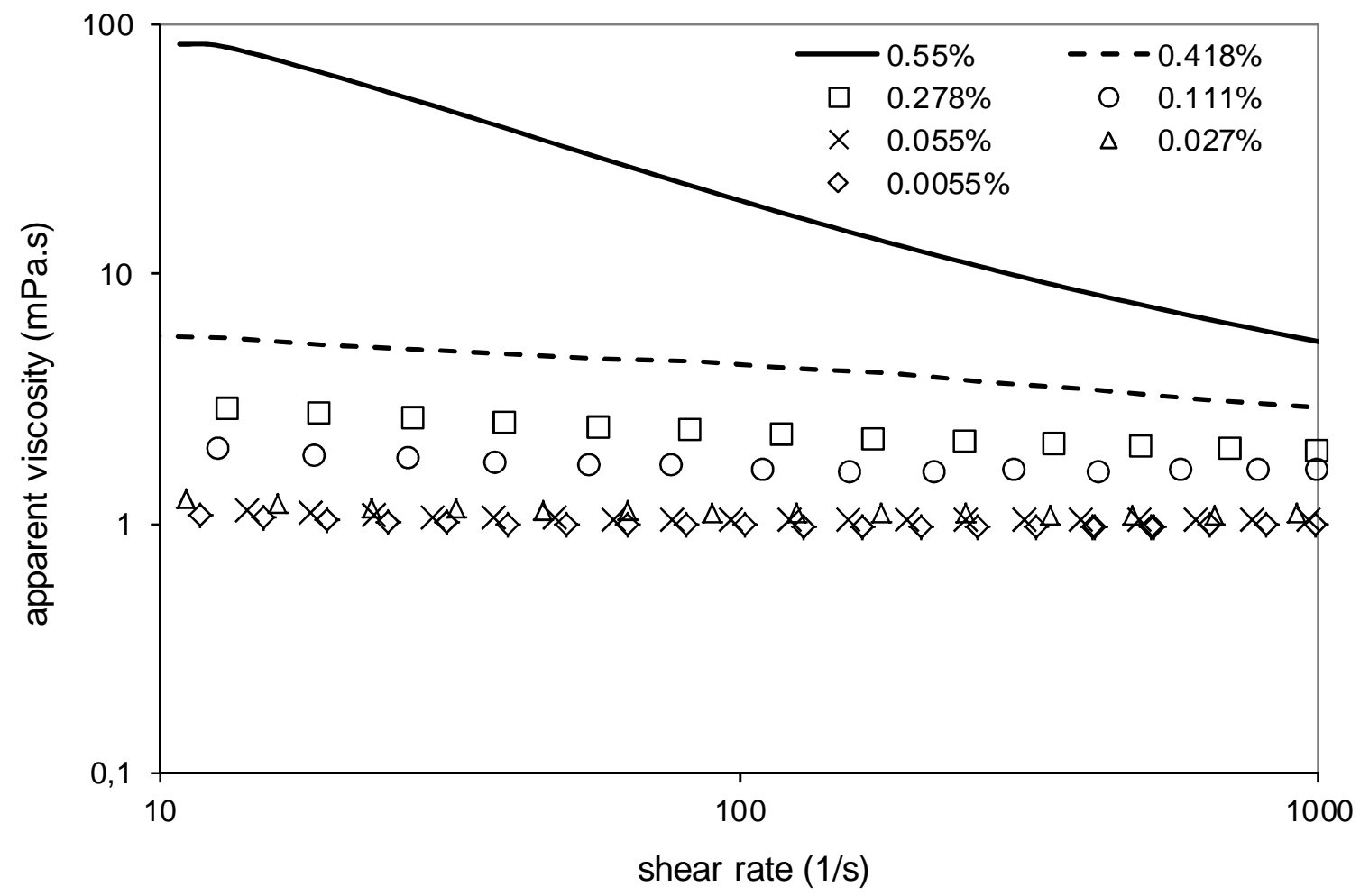

648

649 650

651
Figure 6. Viscosity of nanofluids at $20^{\circ} \mathrm{C}$ as a function of shear rate for different volume fraction of nanotubes. 


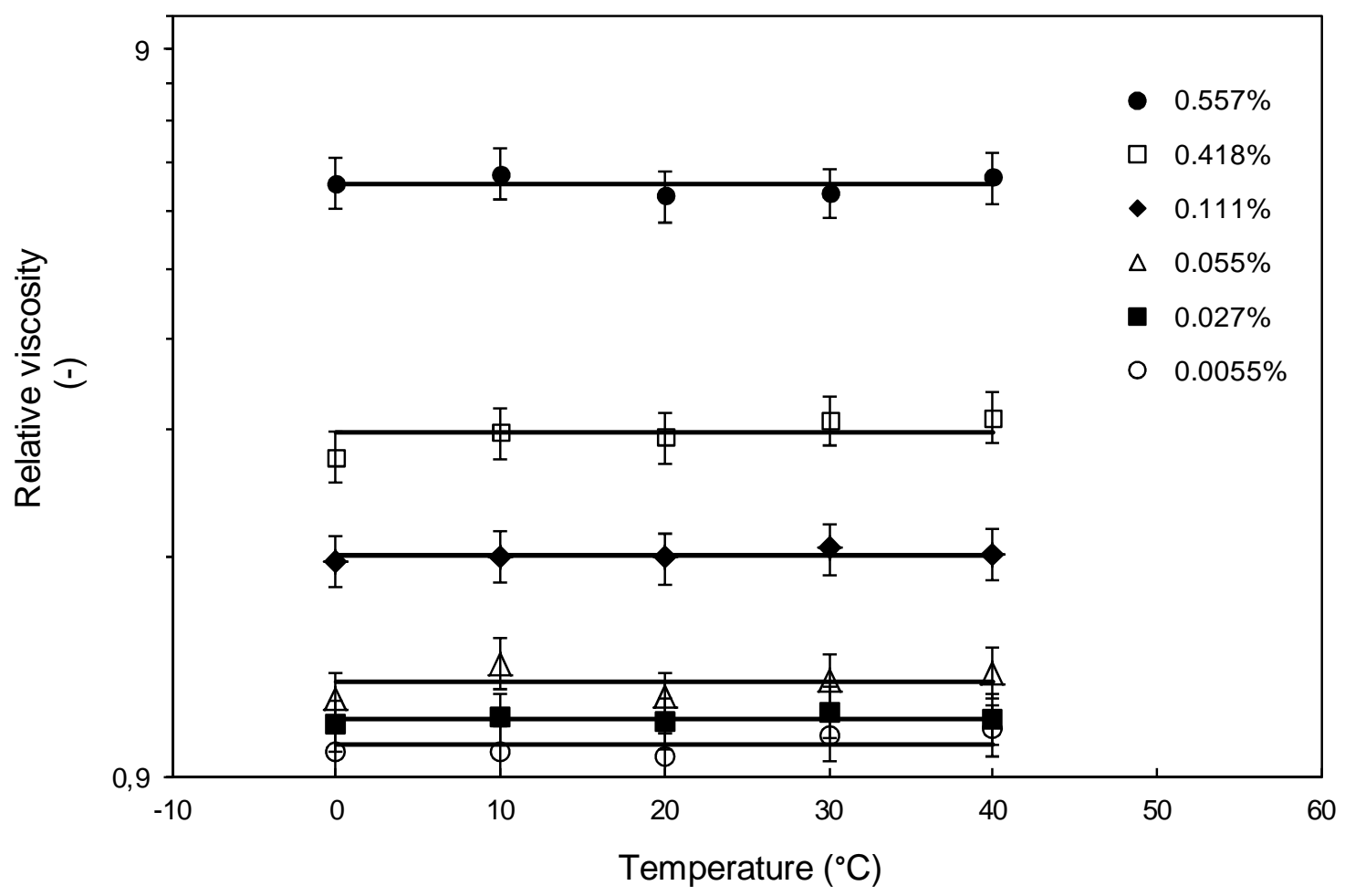

652

653

654 Figure 7. Relative viscosity of nanofluids as a function of particle volume content and 655 temperature. 


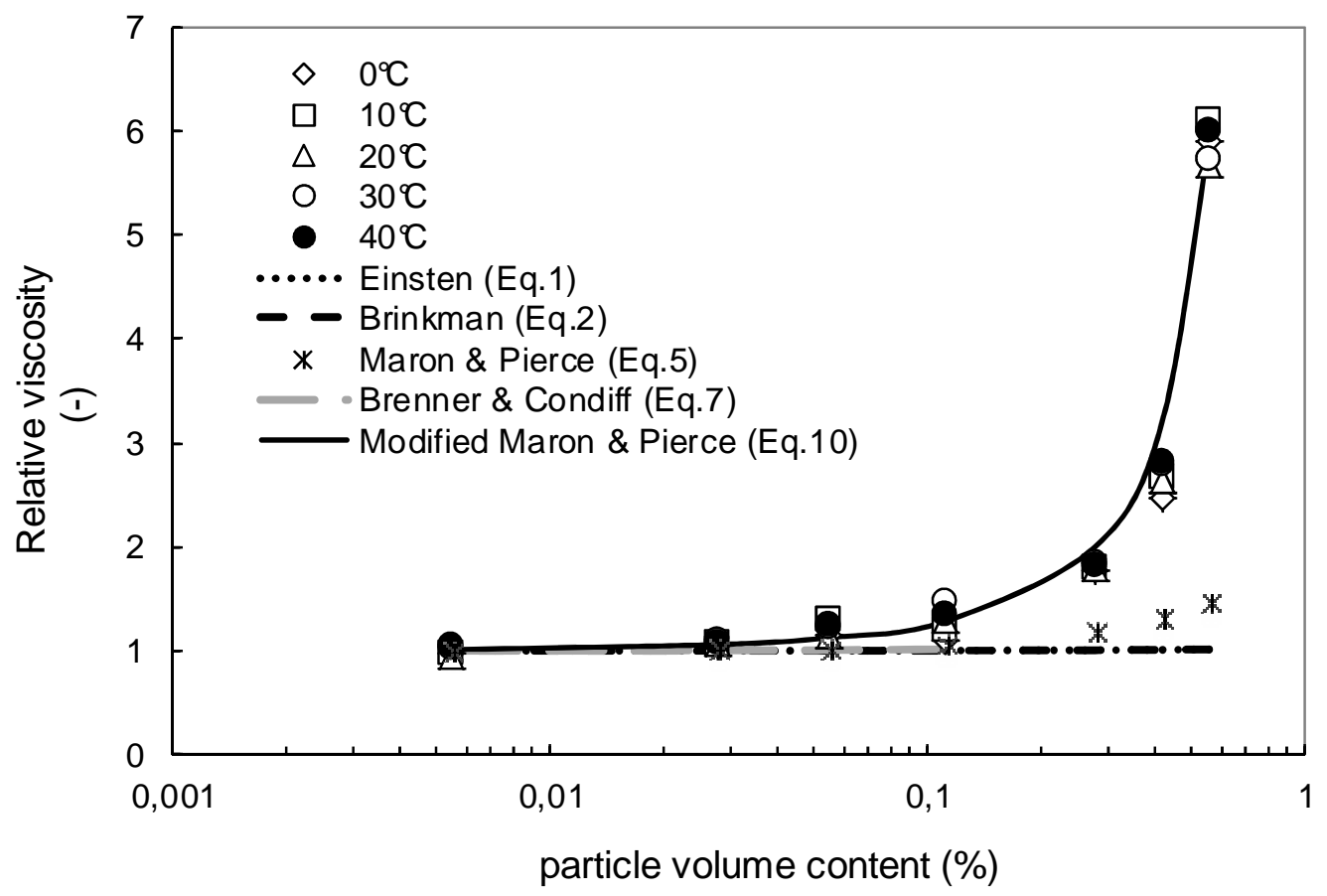

657

658

659

660

Figure 8. Relative viscosity of nanofluids as a function of particle volume content and temperature - Comparison of experimental data and viscosity models. 
661 Table 1. Volume fraction of tested nanofluids and corresponding base fluids, and shear 662 viscosity of base fluids as a function of SDBS volume fraction and temperatures.

663

\begin{tabular}{cc|ccccc}
\hline \multicolumn{2}{c|}{ Volume fraction $(\%)$} & \multicolumn{5}{c}{ Shear viscosity of base fluids $(\mathrm{mPa} . \mathrm{s})$} \\
\hline $\mathrm{CNT}$ & SDBS & $0\left({ }^{\circ} \mathrm{C}\right)$ & $10\left({ }^{\circ} \mathrm{C}\right)$ & $20\left({ }^{\circ} \mathrm{C}\right)$ & $30\left({ }^{\circ} \mathrm{C}\right)$ & $40\left({ }^{\circ} \mathrm{C}\right)$ \\
\hline 0.557 & 1.697 & 1.98 & 1.478 & 1.129 & 0.877 & 0.665 \\
0.418 & 1.272 & 1.975 & 1.454 & 1.102 & 0.852 & 0.657 \\
0.278 & 0.847 & 1.97 & 1.401 & 1.077 & 0.828 & 0.648 \\
0.111 & 0.338 & 1.964 & 1.391 & 1.046 & 0.799 & 0.637 \\
0.055 & 0.169 & 1.962 & 1.386 & 1.036 & 0.789 & 0.633 \\
0.0277 & 0.084 & 1.961 & 1.383 & 1.027 & 0.784 & 0.632 \\
0.0055 & 0.0169 & 1.96 & 1.382 & 1.026 & 0.780 & 0.630 \\
\hline
\end{tabular}

664 